\title{
Homogeneous Systolic Pyramid Automata with $n$-Dimensional Layers
}

\author{
Makoto Sakamoto*, Makoto Nagatomo, Tatsuma Kurogi, Satoshi Ikeda, \\ Masahiro Yokomichi and Hiroshi Furutani \\ Faculty of Engineering, University of Miyazaki, Miyazaki 889-2192, Japan
}

Takao Ito

Institute of Engineering, Hiroshima University, Higashi-Hiroshima, Hiroshima 739-8527, Japan

Yasuo Uchida

Department of Business Administration, Ube National College of Technology,Ube, Yamaguchi 755-8555, Japan

Tsunehiro Yoshinaga

Department of Computer Science and Electronic Engineering, Tokuyama college of Technology, Shunan, Yamaguchi 745-8585, Japan

*Corresponding author, E-mail: sakamoto@cs.miyazaki-u.ac.jp

Tel: +81-985-58-7392, Fax: +81-985-58-7392

\begin{abstract}
Cellular automata were investigated not only in the viewpoint of formal language theory, but also in the viewpoint of pattern recognition. Cellular automata can be classified into some types. A systolic pyramid automata is also one parallel model of various cellular automata. A homogeneous systolic pyramid automaton with $n$-dimensional layers ( $n$-HSPA) is a pyramid stack of $n$-dimensional arrays of cells in which the bottom $n$-dimensional layer (level 0 ) has size an $(a \geq 1)$, the next lowest ( $a-1) n$, and so forth, the (a-1)st $n$-dimensional layer (level $(a-1)$ ) consisting of a single cell, called the root. Each cell means an identical finite-state machine. The input is accepted if and only if the root cell ever enters an accepting state. An $n$-HSPA is said to be a real-time $n$-HSPA if for every $n$-dimensional tape of size $a^{n}(a \geq 1)$ it accepts the $n$-dimensional tape in time $a$-1. Moreover, a 1 - way $n$-dimensional cellular automaton (1-nCA) can be considered as a natural extension of the 1-way two- dimensional cellular automaton to $n$-dimension. The initial configuration is accepted if the last special cell reaches a final state. A 1-nCA is said to be a real- time $1-n C A$ if when started with $n$-dimensional array of cells in nonquiescent state, the special cell reaches a final state. In this paper, we propose a homogeneous systolic automaton with $n$-dimensional layers ( $n$-HSPA), and investigate some properties of real-time $n$-HSPA. Specifically, we first investigate a relationship between the accepting powers of real-time $n$-HSPA's and real-time 1-nCA's. We next show the recognizability of $n$-dimensional connected tapes by real-time $n$-HSPA's.
\end{abstract}

Keywords: cellular automaton, diameter, finite automaton, $n$-dimension, parallelism, pattern recognition, real time.

\section{Introduction and Preliminaries}

The question of whether processing $n$-dimensional digital patterns is much more difficult than $(n-1)$ dimensional ones is of great interest from the theoretical and practical standpoints. Thus, the study of ndimensional automata as a computational model of ndimensional pattern processing has been meaningful[4-
23]. Cellular automata were investigated not only in the viewpoint of formal language theory, but also in the viewpoint of pattern recognition. Cellular automata can be classified into some types [2]. A systolic pyramid automaton is also one parallel model of various cellular automata. In this paper, we propose a homogeneous systolic automaton with $n$-dimensional layers ( $n$-HSPA), and investigate some properties of real-time $n$-HSPA. 
Let $\Sigma$ be a finite set of symbols. An $n$-dimensional tape over $\Sigma$ is an $(n-1)$-dimensional array of elements of $\Sigma$. The set of all $n$-dimensional tapes over $\Sigma$ is denoted by $\Sigma^{(n)}$. Given a tape $x \in \Sigma^{(n)}$, for each $j(1 \leq j \leq n)$, we let $l_{j}(x)$ be the length of $x$ along the $j$ th axis. When $1 \leq i_{j} \leq l_{j}(x)$ for each $j(1 \leq$ $j \leq n)$, let $x\left(i_{1}, i_{2}, \ldots, i_{n}\right)$ denote the symbol in $x$ with coordinates $\left(i_{1}, i_{2}, \ldots, i_{n}\right)$. We concentrate on the input tape $x$ with $l_{1}(x)=l_{2}(x)=l_{3}(x)=\cdots=l_{n}(x)$. A homogeneous systolic pyramid automaton with $n$-dimensional layers $(n-H S P A)$ is a pyramidal stack of $n$-dimensional arrays of cells in which the bottom $n$-dimensional layer (level 0 ) has size $a^{n}$ ( $a \geq 1$ ), the next lowest $(a-1)^{n}$, and so forth, the $(a-1)$ st $n$-dimensional layer (level $(a-1)$ ) consisting of a single cell, called the root . Each cell means an identical finite-state machine, $M=(Q, \Sigma$, $\delta$, \#, F), where $Q$ is a finite set of states, $\Sigma \subseteq Q$ is a finite set of input states, $\# \in Q-\Sigma$ is the quiescent state, $F \subseteq Q$ is the set of accepting states, and $\delta: Q^{2^{n}+1} \rightarrow Q$ is the state transition function, mapping the current states of $M$ and its $2^{n}$ son cells in a $2 \times 2 \times \cdots \times 2$ block on the $n$-dimensional layer below into M's next state. The input is accepted if and only if the root cell ever enters an accepting state. An $n-H S P A$ is said to be a real-time $n$-HSPA if for every $n$-dimensional tape of size $a^{n}(a \geq 1)$ it accepts the $n$-dimensional tape in time $a-1$. By $£^{R}[n-H S P A]$ we denote the class of the sets of all the $n$-dimensional tapes accepted by a real-time $n$-HSPA[1]. A 1-way $n$-dimensional cellular automaton (1-nCA) can be considered as a natural extension of the 1-way two-dimensional cellular automaton to $n$ dimensions [3]. The initial configuration of the cellular automaton is taken to be an $l_{1}(x) \times l_{2}(x) \times \cdots \times l_{n}(x)$ array of cells in the nonquiescent state. The initial configuration is accepted if the last special cell reaches a final state. A 1-nCA is said to be a real-time $1-n C A$ if when started with an $l_{1}(x) \times l_{2}(x) \times \cdots \times l_{n}(x)$ array of cells in the nonquiescent state, the special cell reaches a final state in time $l_{1}(x)+l_{2}(x)+\cdots+l_{n}(x)-1$. By $£^{R}[1-n C A]$ we denote the class of the sets of all the $n$-dimensional tapes accepted by a real-time 1-nCA [3].

\section{Main Results}

We mainly investigate a relationship between the accepting powers of real-time $n$-HSPA's and real-time 1-nCA's. The following theorem implies that real-time $n$-HSPA's are less powerful than real-time 1-nCA's.

Theorem 2.1. $£^{R}[n-H S P A] \subsetneq £^{R}[1-n C A]$.

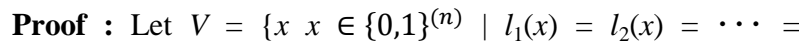
$l_{n}(x) \&\left[\forall i_{1}, \forall i_{2}, \ldots, \forall i_{n-1}\left(1 \leq i_{1} \leq l_{1}(x), 1 \leq i_{2} \leq l_{2}(x), \ldots, 1\right.\right.$ $\left.\left.\left.\leq i_{n-1} \leq l_{n-1}(x)\right)\left[x\left(i_{1}, i_{2}, \ldots, i_{n-1}, 1\right)=x\left(i_{1}, i_{2}, \ldots, i_{n-1}, l_{n}(x)\right)\right]\right]\right\}$.

It is easily shown that $V_{1} \in £^{R}[1-n C A]$. Below, we show that $V \notin £^{R}[n-H S P A]$. Suppose that there exists a real-time $n$-HSPA $(n=3)$ accepting $V$. For each $t \geq 4$, let

$W(n)=\left\{x \in\{0,1\}^{(3)} \mid l_{1}(x)=l_{2}(x)=\cdots=l_{n}(x) \&[x(1,2,1)\right.$, $\left.(t, t-1, t)] \in\{0\}^{(3)}\right\}$.

Eight sons of the root cell $A_{(t-1,1,1,1)}$ of $M A_{(t-2,1,1,2)}, A_{(t-2,1,2,2)}$, $A_{(t-2,2,1,2)}, A_{(t-2,2,2,2)}, A_{(t-2,1,1,3)}, A_{(t-2,1,2,3)}, A_{(t-2,2,1,3)}, A_{(t-2,2,2,3)}$ are denoted by $C_{U N W}, C_{U S W}, C_{U S E}, C_{U N E}, C_{D N W}, C_{D S W}, C_{D S E}, C_{D N E}$, respectively. For each $x$ in $W(n), x(U N W), x(U S W), x(U S E)$, $x(U N E), x(D N W), x(U S W), x(U S E), x(U N E)$ are the states of CUNW, CUSW, CUSE, CUNE, CDNW, CDSW, CDSE, CDNE, at time $t-2$, respectively. Let $\sigma(x)=(x(U N W), x(U S W)$, $x(D N W), \quad x(D S W)), \quad \gamma(x)=(x(U S E), \quad x(U N E), \quad x(D S E)$, $x(D N E))$. and $\rho(x)=(x(U N W), x(U S W), x(D N W), x(D S W)$, $x(U S E), x(U N E), x(D S E), x(D N E))$. Then, the following two propositions must hold:

Proposition 2.1. (i) For any two tapes $x, y \in W(n)$ whose 1 st $(1-3)$ planes are same, $\sigma(x)=\sigma(y)$. (ii) For any two tapes $x, y \in W(n)$ whose $n$-th(1-3) planes are same, $\gamma(x)=$ $\gamma(y)$

[Proof : From the mechanism of each cell, it is easily seen that the states of $C_{U N W}, C_{U S W}, C_{D N W}, C_{D S W}$ are not influenced by the information of $x(1-3)_{t}$ 's. From this fact, we have (i). The proof of (ii) is the same as that of (i). $\square]$

Propositon 2.2. For any two tapes $x, y \in W(t)$ whose $1 s t$ (1-3) planes are different, $\sigma(x) \neq \sigma(y)$.

[Proof : Suppose to the contrary that $\sigma(x)=\sigma(y)$. We consider two tapes $x^{\prime}, y^{\prime} \in W(t)$ satisfying the following :

(i) $x(1-3)_{1}$ and $x(1-3)_{t}$, are equal to $x(1-3)_{1}$ of $x$, respectively

(ii) $y^{\prime}(1-3)_{1}$ is equal to $y(1-3)_{1}$, and $y^{\prime}(1-3)_{t}$ is equal to $x(1$ $-3)_{1}$.

As is easily seen, $x^{\prime} \in V$ and so $x^{\prime}$ is accepted by $M$. On the other hand, from Proposition 2.1(ii), $\gamma\left(x^{\prime}\right)=\gamma\left(y^{\prime}\right)$. From Proposition 2.1(i), $\sigma(x)=\sigma\left(x^{\prime}\right), \sigma(y)=\sigma\left(y^{\prime}\right)$. It follows that $y^{\prime}$ must be also accepted by $M$. This contradicts the fact that $y^{\prime}$ is not in $V$. $\square]$ 
Proof of Theorem 2.1 (continued) : Let $p(t)$ be the number of tapes in $W(t)$ whose 1 st (1-3) planes are different, and let $Q(t)$ $=\{\sigma(x) \mid x \in W(t)\}$, where $\mathrm{k}$ is the number of states of each cell of $M$. Then, $p(t)=2^{t^{2}}$, and $Q(t) \leq k^{4}$. It follows that $p(n)$ $>Q(t)$ for large $t$. Therefore, it follows that for large $t$, there must be two tapes $x, y$ in $W(t)$ such that their 1st (1-3) planes are different and $\sigma(x)=\sigma(y)$. This contradicts Proposition 2.2, so we can conclude that $V \notin £^{R}[3-H S P A]$. In the case of $n$-dimension, we can show that $V \notin £^{R}[n$-HSPA] by using the same technique. This completes the proof of Theorem 2.1.

We next show the recognizability of n-dimensional connected tapes by real-time $n$-HSPA's by using the name technique of Ref.[3]. Let $x$ in $\{0,1\}^{(n)}$. A maximal subset $P$ of $\boldsymbol{N}^{n}$ satisfying the following conditions is called a 1-component of $x$.

(i)For any $\left(i_{1}, i_{2}, \quad \ldots \quad, i_{n} \in P\right.$, we have $1 \leq i_{1} \leq l_{1}(x)$, $1 \leq i_{2} \leq l_{2}(x), \ldots, 1 \leq i_{n} \leq l_{n}(x)$, and $x\left(i_{1}, i_{2}, \ldots, i_{n}\right)=1$.

(ii) For any $\left(i_{1}, i_{2}, \ldots, i_{n}\right),\left(i_{1}{ }^{\prime}, i_{2}, \ldots, i_{\mathrm{n}}{ }^{\prime}\right) \in P$, there exists a sequence $\left(i_{1,0}, i_{2,0}, \ldots, i_{n, 0}\right),\left(i_{1,1}, i_{2,1}, \ldots, i_{n, 1}\right), \ldots$, $\left(i_{1}, i_{2}, \ldots, \ldots, i_{n, n}\right)$ of elements in $P$ such that $\left(i_{1,0}, i_{2}, 0, \ldots, i_{n, 0}\right)=$ $\left(i_{1}, i_{2}, \ldots, i_{n}\right),\left(i_{1}, n, i_{2}, n, \ldots, i_{n, n}\right)=\left(i_{1}^{\prime}, i^{\prime}{ }_{2}, \ldots, i_{n}^{\prime}\right)$, and $\left|i_{1, j}-i_{1, j-1}\right|$ $+\left|i_{2, j}-i_{2, j-1}\right|+\ldots+\left|i_{n, j}-i_{n, j-1}\right| \leq 1(1 \leq j \leq n)$. A tape $x \in\{0$, $1\}^{(n)}$ is called connected if there exists exactly one 1component of $x$

Let $T_{c}$ be the set of all the $n$-dimensional connected tapes. Then, we have

Theorem 2.2. $T_{c} \notin £^{R}[n-H S P A]$.

\section{Conclusion}

We investigated a relationship between the accepting powers of homogeneous systolic pyramid automaton with $n$-dimensional layers $(n-H S P A)$ and one-way $n$-dimensional cellular automata (1-nCA) in real time, and showed that real-time $n$-HSPA's are less powerful than real time 1-nCA's.

\section{References}

1. K.Culik II, J.Gruska and Salomaa, Sytolic trellis automata, Part I, International Journal of Computer Mathmatics, 55:pp.99-121, 1991.

2. C. Iwamoto, K.Inoue, and I.Takanami, Some Properties of Homogeneous Pyramid Automata, The IEICE Transactions on Information and Systems (Japanese Edition), J73-D-I(9) : 778-780, 1990.

3. C. Iwamoto, K. Inoue, and I. Takanami, Some Properties of Homogeneous Pyramid Automata, The IEICE
Transactions on Information and Systems (Japanese Edition), J73-D-I(9) : 778- 780, 1990.

4. K. Krithivasan and M. Mahajan, Systolic pyramid automata, cellular automata and array languages, International Journal of Pattern Recognition and Artificial Intelligence, 3(3 4):405-433, 1989.

5. M.Sakamoto, Three-Dimensional Alternating Turing Machines, Ph.D. Thesis, Yamaguchi University, 1999.

6. M.Sakamoto, H.Okabe, and K.Inoue, Some properties of four-dimansional finite automata, in 2002 Chugoku Section Joint Convention Record of Insistes of Electrical and Information Engineerings, Japan, p.351,2002.

7. M.Sakamoto, S.Nagami, K.Kono, A relation- ship between the accepting powers of three- dimentional layers, Trans. of SCI(Japan), Vol.17, No.10, pp.451-458,2004.

8. M.Sakamoto, H.Okabe, S.Nagami, S.Taniguchi, T.Maki, Y.Nakama, M.Saito, M.Kono, and K.Inoue, A note on four-dimensional finite au- tomata, WSEAS Transactions on Computers, Issue 5,Vol.3, pp.1651-1656,2004.

9. M.Sakamoto, T.Ito, N.Tomozoe, and H.Furutani, Asurvey of three-dimensional automata, The papers of Technical Meeting on Information Systems, IEE, Japan, IS-07-12, pp.11-17,2007.

10. M.Sakamoto, T.Ito, H.Furutani, and M.Kono, Some accepting powers of three-dimensional parallel Turning machines, in 1st European Workshop on Artificial Life and Robotics, V ienna, Austria, pp.73-79,2007.

11. M.Sakamoto, N.Tomozoe, H.Furutani, M.Kono, T.Ito, Y.Uchida, and H.Okabe, A survey of automata on three-dimensional input tapes, WSEAST Transactions on Computers,Issue 10,Vol.7, pp.1638-1647,2008.

12. M.Sakamoto, T.Ito, H.Furutani, and M.Kono, Some accepting powers of three-dimensional parallel Turning machines, International Journal of Artificial Life and Robotics,Vol.13,No.1, pp.27-30,2008.

13. M.Sakamoto, M.Fukuda, S.Okatani, T.Ito, H.Furutani, and M.Kono, Path-bouded Three- dimensional finite automata, International Journal of Artificial life and Robotica, Springer,Vol.13,No.1,pp.54-57,2008.

14. M.Sakamoto, S.Okatani, M.Fukuda, T.Ito, and H.Furutani, and M.Kono, A relationship between Turning machines and finite automata on four-dimensional input tapes, International Journal of Artificial life and Robotica, Springer, Vol.13,No.1, pp.58-60,2008.

15. M.Sakamoto, S.Okatani, K.Kajisa, M.Fukuda, T.Matsuawa, A.Taniue, T.Ito, H.Furutani, and M.Kono, Hierarchies based on the number of cooperating systems of three-dimensional finite automata,International Journal of Artificial Life and Robotics,Vo.4,No.3, pp.425-428.2009.

16. M.Sakamoto, T.Matsukawa, R.Katamune, H.Furutani, M.Kono, S.Ikeda, T.Ito, Y.Uchida, and T.Yoshinaga, Four-dimensional synchronized alternating Turning machines,Proceedings of the 10th American Conference 
on Applied Mathmatics, Harvard University, Cambridge, USA, pp.195-200,2010(CD-ROM).

17. M.Sakamoto, R.Katamune, T.Matsukawa, H.Furutani, M.Kono, S.Ikeda, T.Ito, Y.Uchida, and T.Yoshinaga, Some result about hierarchy and recognizability of four-dimensional sychronized alternating Turning machines, Proceedings of the 10th American Conference on Applied Mathmatics, Harvard University, Cambridge, USA, pp.201-205, 2010(CD-ROM).

18. M.Sakamoto, T.Matsukawa, R.Katamune, H.Furutani, M.Kono, S.Ikeda, T.Ito, Y.Uchida, and T.Yoshinaga, Synchronized Alternating Turning Machines on Four-Dimensional In- put Tapes, WSES TRANSACTIONS on COMPUTERS,Issue 4,Vol.9, pp.319-328,2010.

19. M.Sakamoto, R.Katamune, T.Matsukawa, H.Furutani, M.Kono, S.Ikeda, T.Ito, Y.Uchida, and T.Yoshinaga, Hardware Hierarchies and Recognizabilities of Four-Dimensional Synchro- nized Alternating Turning Machines, WSES TRANSACTIONS on COMPUTERS,Issue 4. pp.329-338,2010.

20. M.Sakamoto, T.Ito, X.Qingquan, Y.Uchida, T.Yochinaga, M.Yokomichi, S.Ikeda, and H.Furutani, A Note on Three-dimensional Prob- abilistic Finite Automata, The Seventeenth International Symposium on Artificial Life and Robotics 2012, Oita, Japan, pp.492495,2012(CD-ROM).

21. M.Sakamoto, Y.Uchida, T.Hamada, T.Ito, T.Yoshinaga, M.Yokomichi, S.Ikeda, and H.Furutani, A Relationship between the Accept- ing Powers of Nondeterministic Finite Automata and Probabilistic Finite Automata on Three- Dimensional Input Tapes, in the 2012 IEICE General Conference, Okayama University, p.4,2012(CD-ROM).

22. T. Toffoli and N. Margolus, Cellullar automaton machines - new environment for modeling, MIT Press, 1987.

23. J. Wiedermann, Parallel Turing machines, Technical Report RUU-CS-84-11, Department of Computer Science, University of Utrecht, the Netherlands, 1984. 